\title{
Self-similarity in the classification of finite-size scaling functions for toroidal boundary conditions
}

\author{
Tsong-Ming Liaw, ${ }^{1, *}$ Ming-Chang Huang, ${ }^{2, \dagger}$ Yu-Pin Luo, ${ }^{3}$ Simon C. Lin, ${ }^{1}$ Yen-Liang Chou, ${ }^{4}$ and Youjin Deng ${ }^{5}$ \\ ${ }^{1}$ Grid Computing Centre and Institute of Physics, Academia Sinica, 11529 Taipei, Taiwan \\ ${ }^{2}$ Center for Nonlinear and Complex Systems and Department of Physics, Chung-Yuan Christian University, 32023 Chungli, Taiwan \\ ${ }^{3}$ Physics Division, National Center for Theoretical Science, Hsinchu 30013, Taiwan \\ ${ }^{4}$ Department of Physics, Virginia Tech. Blacksburg, Virginia 24061-0435, USA \\ ${ }^{5}$ Physikalisches Institut, Universität Heidelberg, Philosophenweg 12, 69120 Heidelberg, Germany
}

(Received 4 May 2007; published 2 January 2008)

\begin{abstract}
The conventional periodic boundary conditions in two dimensions are extended to general boundary conditions, prescribed by primitive vector pairs that may not coincide with the coordinate axes. This extension is shown to be unambiguously specified by the twisting scheme. Equivalent relations between different twist settings are constructed explicitly. The classification of finite-size scaling functions is discussed based on the equivalent relations. A self-similar pattern for distinct classes of finite-size scaling functions is shown to appear on the plane that parametrizes the toroidal geometry.
\end{abstract}

DOI: 10.1103/PhysRevE.77.010101

Finite-size scaling theory, initiated more than three decades ago by Fisher and Barber [1], concerns itself with how the thermodynamic behaviors of finite systems are modified near critical points in response to the onset of large fluctuations. Accordingly, the occurrence of rounding of the critical point singularity in the thermodynamic response functions can be expressed in terms of the finite-size scaling functions (FSSFs) $[2,3]$. The FSSFs of a variety of quantities were determined for different lattice structures and boundary conditions (BCs) in the earlier studies [4-13]. The analyses have enhanced our understanding of the scaling behaviors of finite systems. In particular, the relations among the critical amplitudes have provided more stringent tests of the universality class to which finite systems belong [2]. Moreover, noticeable properties of FSSFs also have been revealed in the investigations. For example, it was shown that universal FSSFs of different lattice structures with the conventional periodic BCs can be achieved by adjusting the aspect ratios and the metric factors [13]. In general, FSSFs are very sensitive to the aspect ratio and BCs. The classification of FSSFs for systems on a specified planar lattice is mainly a geometric issue with the significance incorporated essentially in various BC settings. This Rapid Communication is devoted to a complete classification of FSSFs for planar lattice systems with full toroidal BCs, a generalization of the conventional periodic BCs. Our endeavor is to establish the transformation laws which determine the equivalent geometric structures among systems of fixed area. Such attempts have been made by Okabe et al. [6] and Ziff et al. [7]. The present study provides a systematic and complete treatment of this issue. Moreover, as a consequence of equivalent geometric structures, we report the appearance of a fractal pattern formed by the regions corresponding to distinct classes of FSSFs on the parametrization plane for full toroidal BCs.

The general prescription for the full class of periodic BCs on planar lattices, subject to the doubly connected topology, is based on pairs of primitive vectors. As shown in Fig. 1(a),

\footnotetext{
*1tming@gate.sinica.edu.tw

†ming@phys.cycu.edu.tw
}

PACS number(s): 64.60.A-, 05.50.+q, 75.10.Hk

primitive vectors are linearly independent vectors which separately preimpose the identifications of boundary variables [14]. When the two primitive vectors are mutually perpendicular, the specified geometry is a helical torus, in the context of nanotube physics [15], labeled by the chirality $\chi$ as depicted in Fig. 1(b). Hence, the conventional periodic $\mathrm{BCs}$ in which both vectors coincide with the coordinate axes correspond to the helical ones with trivial chirality, $\chi=0$. However, distinct pairs of primitive vectors may end up with the same period identification if they are related by a matrix $\mathcal{M}$,

$$
\left(\begin{array}{l}
\vec{a}_{1}^{\prime} \\
\vec{a}_{2}^{\prime}
\end{array}\right)=\mathcal{M}\left(\begin{array}{l}
\vec{a}_{1} \\
\vec{a}_{2}
\end{array}\right) \quad \text { with } \operatorname{det}(\mathcal{M})=1,
$$

where the matrix $\mathcal{M}$ has integer elements, and the condition for the unity of the determinant is to ensure the same area of the unit cell. This is known to be an $\operatorname{SL}(2, Z)$ transform, the prototype of modular symmetry discussed in the context of conformal field theory [16]. We notice that any sign flipping [7] and/or reordering of the primitive vectors, which may not be allowed by $\operatorname{SL}(2, Z)$ transforms, does not alter the setting of boundary conditions.

There exists another prescription of periodic BCs called the twist setting [6-8] for which, as shown in Fig. 2(a), the direction of one of the primitive vectors is kept fixed, and it may coincide with one of the lattice orientations. Note that the terminology used in the literature has aroused some con-

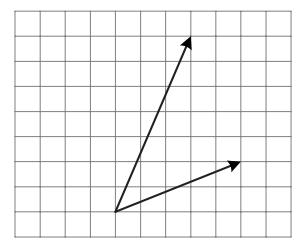

(a)

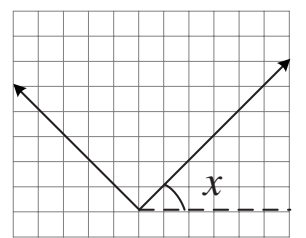

(b)
FIG. 1. Two primitive vectors on a planar square lattice: (a) general case; (b) the orthogonal case with the deviation from the coordinate axes of the underlying lattice by an angle $\chi$ called chirality. 


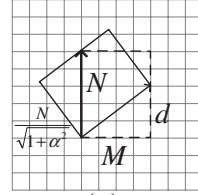

(a)

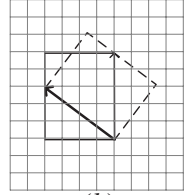

(b)

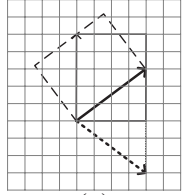

(c)
FIG. 2. (a) Twisted BC specified by the primitive vectors for the aspect ratio $A=N / M$ and the twisting factor $\alpha=d / M$. The vectors are rotated counterclockwise with the angle $\pi / 2-\arctan (\alpha)$ to another set, shown in (b). By reversing one vector followed by $\alpha \rightarrow-\alpha$, we achieve the vectors shown in (c), which gives rise to the new aspect ratio $\left(1+\alpha^{2}\right) / A$ and leaves $\alpha$ unaltered.

fusions. The term twisting has been used as synonymous with antipode [10]; however, no conflict arises here when restricted to the toroidal geometry. Moreover, it has also been referred to simply as helical, a usage different from that of particular significance for chiral nanotubes [15]. Henceforth, we shall employ the term twisting as indicating the particular choice of primitive pairs described above, and the term fully helical for tori equipped with definite chirality. We notice that the twisting prescription is equivalent to the general prescription. This can be proved by showing that a map of the general prescription onto the twisting prescription via an $\mathrm{SL}(2, Z)$ transform is always achievable, as given explicitly in the following.

Consider a planar square lattice with a general periodic BC prescribed by $\vec{a}_{1}=x_{1} \hat{x}+y_{1} \hat{y}$ and $\vec{a}_{2}=x_{2} \hat{x}+y_{2} \hat{y}$, where $\hat{x}$ and $\hat{y}$ are unit vectors specifying the lattice orientation. Based on Eq. (1), we can transform this to an $M \times N$ lattice with a twisted $\mathrm{BC}$, specified by $\vec{b}_{1}=M \hat{x}+d \hat{y}$ and $\vec{b}_{2}=N \hat{y}$, via the transformation matrix,

$$
\mathcal{M}=\left(\begin{array}{cc}
m_{1} & m_{2} \\
-x_{2} / \operatorname{GCD}\left(x_{1}, x_{2}\right) & x_{1} / \operatorname{GCD}\left(x_{1}, x_{2}\right)
\end{array}\right),
$$

where $m_{1}$ and $m_{2}$ are determined by $m_{1} x_{1}+m_{2} x_{2}$ $=\operatorname{GCD}\left(x_{1}, x_{2}\right)$, and $\operatorname{GCD}\left(x_{1}, x_{2}\right)$ is the greatest common divisor of $x_{1}$ and $x_{2}$. Accordingly, $M=\operatorname{GCD}\left(x_{1}, x_{2}\right), N=\left(x_{1} y_{2}\right.$ $\left.-x_{2} y_{1}\right) / M$, and $d=m_{1} y_{1}+m_{2} y_{2}$. Note that as a special case of Eq. (2) the matrix $\mathcal{M}$ for the unique transformation of a fully helical torus to the twisted $\mathrm{BC}$ has been explicitly determined in Ref. [8]. Hence, a twist setting suffices for complete characterization of toroidal BCs; we then define the BC-relevant parameters as the aspect ratio $A=N / M$ and the twisting factor $\alpha=d / M$ as depicted in Fig. 2(a). Subsequently, on this basis we exploit the transformation laws among equivalent geometric structures.

The transformation of reversing the twisting factor, $\alpha \rightarrow-\alpha$, corresponds to a change of twisting of the boundary tori from clockwise to counterclockwise, or vice versa, and this is indistinguishable for the partition function of a physical system. In addition, as a symmetry of $\operatorname{SL}(2, Z)$, the transformation of changing $\alpha$ by additional integer multiples of the aspect ratio, $\alpha \rightarrow s A+\alpha$, also leaves the system boundary unmodified. The two equivalent transformations above indicate that the subspace with parameter range $0<\alpha \leq A / 2$ satisfies the complete characterization of the general periodic
BCs. Furthermore, one may expect to have rotational invariance for isotropic systems. By combining this with other equivalent transformations, we obtain another invariant relation, namely, $A \rightarrow\left(1+\alpha^{2}\right) / A$, with $\alpha$ kept fixed, as shown explicitly in Figs. 2(a)-2(c).

An immediate test is performed by applying the equivalent relations to FSSFs. The Binder parameters [12], defined as $g(m)=1-\left\langle m^{4}\right\rangle / 3\left\langle m^{2}\right\rangle^{2}$, for the two-dimensional (2D) $q$-state Potts models with $q=2$ and 3 , are measured by the Monte Carlo method [17]. Here, the critical temperature is known to be $T_{c}=1 / \ln (1+\sqrt{q})[18]$ and the Potts magnetization density $m$ is defined via $m^{2}=\sum_{i=0}^{q-2} \sum_{j=i+1}^{q-1}\left(\rho_{i}-\rho_{j}\right)^{2} /(q-1)$ with $\rho_{i}$ for the fraction of lattice sites associated with the state $i$. The definitions above yield $g(m) \rightarrow 2 / 3$ and $g(m) \rightarrow 2(q-2) /[3(q-1)]$ for the limits of low and high temperature, respectively. The simulation results, plotted as $g(m)$ versus $L^{1 / \nu}\left(T-T_{c}\right) / T_{c}$ with $\nu=1$, for sets of lattices related by the transformations $\alpha \rightarrow s A \pm \alpha$ with $A$ unaltered, are shown in Fig. 3(a); similarly, the results for those related by $A \rightarrow\left(1+\alpha^{2}\right) / A$ upon fixing $\alpha$ are shown in Fig. 3(b). Here $L$ is defined as the square root of the area of a primitive cell. All results agree completely with our assertion. Thus, we may conclude that the BCs equipped with a definite FSSF are related by composite operations of

$$
(A, \alpha) \rightarrow(A, s A \pm \alpha), \quad \forall s \in Z,
$$

and

$$
(A, \alpha) \rightarrow\left(\frac{1+\alpha^{2}}{A}, \alpha\right) .
$$

We notice that the invariant aspect ratio $A /\left(1+\alpha^{2}\right)$, which was conjectured in Ref. [6] based on the symmetry of rotation, coincides with the chiral aspect ratio $B$ [19] only for $\chi=\alpha$; on the other hand, the symmetry specified by Eq. (7) of Ref. [7] can be shown to be equivalent to Eq. (4) on our parametrization plane.

The finite-size scaling behaviors, in general, anticipate more accordance among distinct systems, since only the critical region and the large-size limit are concerned. Direct numerical observations suggest that for $A \leq 1$ the difference among the twisting factors $\alpha$ becomes immaterial for the FSSFs. For definiteness, two concrete examples of different measurements are given as follows. The critical shift $\theta$, $\theta=\left(T_{\max }-T_{c}\right) / T_{c}$, measures the deviation of the specific heat peak $T_{\max }$ from the critical temperature $T_{c}$. For the $2 \mathrm{D}$ Ising model, the critical shift scales with system size $L$ as $\theta \sim c / L$, where the amplitude $c$ is expected to depend on the values of $A$ and $\alpha$. However, the numerical results based on the exact form of the partition function tend to suggest that the $c$ values are independent of $\alpha$ for $A \leq 1$ [8]. The other example is the simulation data of the $2 \mathrm{D} q$-state Potts models with $q=2$ and 3, shown as $g(m)$ versus $L\left(T-T_{c}\right) / T_{c}$ in Fig. 4; the results for the same $A$ but different $\alpha$ values coalesce into a single curve for $A \leq 1$.

This fact, namely, FSSFs of different $\alpha$ values are almost indistinguishable for any given $A$ value below the threshold $A=1$, denotes a kind of analytic continuation in specifying the classes of FSSFs for $A \leq 1$; this can be employed to ana- 


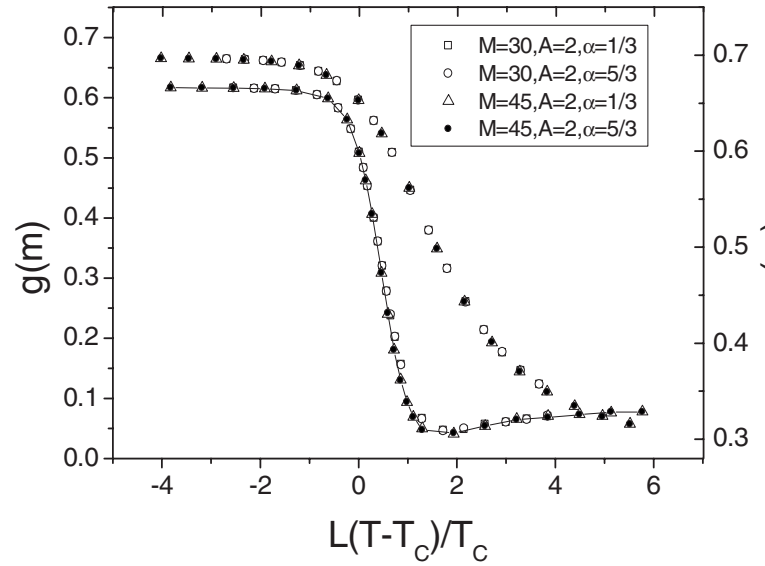

(a)

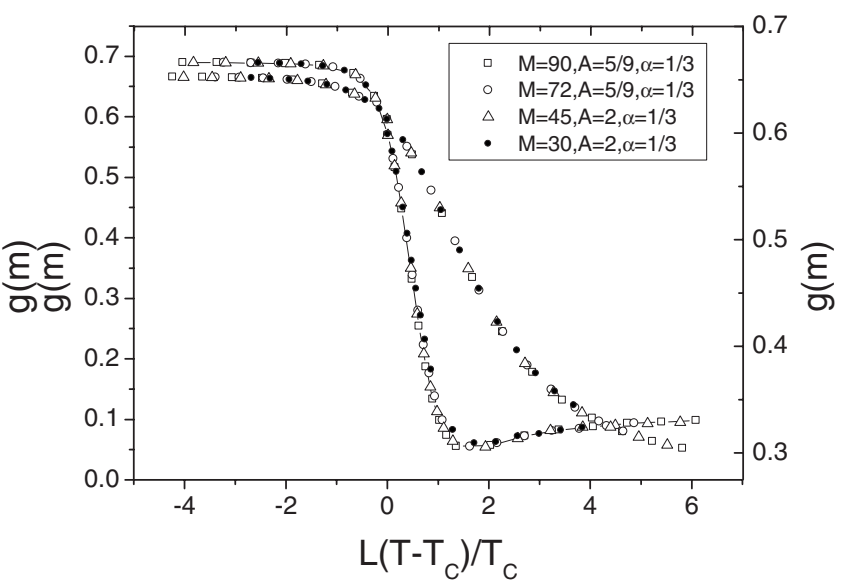

(b)

FIG. 3. Binder parameter $g(m)$ versus $L\left(T-T_{c}\right) / T_{c}$ for different lattices related by the transformation (a) $\alpha \rightarrow s A \pm \alpha$ with $A$ kept fixed and (b) $A \rightarrow\left(1+\alpha^{2}\right) / A$ with $\alpha$ kept fixed. The left (right) vertical scale is for $q=2$ (3), the data are connected with a smooth solid line for $q=3$.

lytically continue the parametrization plane of $A$ and $\alpha$ to a real space. The continuous curves shown in Fig. 5 are the results of applying Eqs. (3) and (4) sequentially to the initial geometries on the horizontal line $A=1$; these then share the same FSSFs. Note that the transformations will not cause any irrational counterpart on the parametrization plane since none of the transformations will transform rational coordinates into irrational ones, and vice versa. Some remarks on Fig. 5 are as follows. (1) The intersections between the parabolic curves, marked by the circles, represent a class of helical tori of unity aspect ratio, $B=1$. Certainly, chirality may vary within the $B=1$ set, since rotation is involved in the transformation of Eq. (4). (2) The curves are the boundaries of two distinctive regions $R_{1}$ and $R_{2}$ with $R_{2}$ for the domains enclosed by the curved triangles and $R_{1}$ for the rest, as depicted in Fig. 5. No transformations based on Eqs. (3) and (4) are found relating $R_{1}$ and $R_{2}$. (3) The geometries for other distinct classes of FSSFs can be obtained by using different horizontal lines of $A<1$ as the initial geometries; further sequential transformations yield other members which be- long to the same class and are located at the subdomains of $R_{1}$. (4) The transformations of the point $(A, \alpha)$ with $A>1$ yield a close contour in $R_{2}$ ranging over limited $\alpha$ values. Note that no parametrization of BCs within $R_{2}$ can be rendered fully helical by means of Eq. (1).

The self-similarity for the pattern of a distinct class of FSSFs may appear more transparently when the new set of parameters $u=\alpha / A=d / N$ and $v=1 / A$ is adopted. Here, the parameter $u$ has also been referred to as tilting [7] and $v$ inversely defines the aspect ratio. In the new parametrization plane, the pattern exhibits periodicity with unity period in the $u$ direction and self-similarity in the $v$ direction toward the origin. In Fig. 6, two distinct classes of FSSFs, corresponding to $A=1$ and 0.5 , are drawn on the $u-v$ plane. The results for $A=1$, marked by crosses in Fig. 6, show that the contours can be viewed as circles with the circles further inserted between two other circles in the $v$ direction toward the origin and so on, remarkably resembling the Apollonian gasket [20]. Accordingly, the correspondence between Figs. 5 and 6 can be established as follows. The region $R_{1}$ becomes the

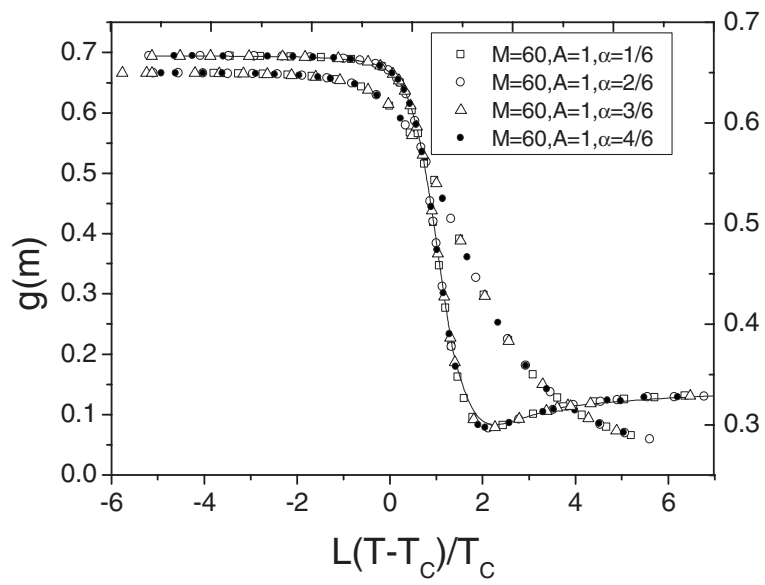

(a)

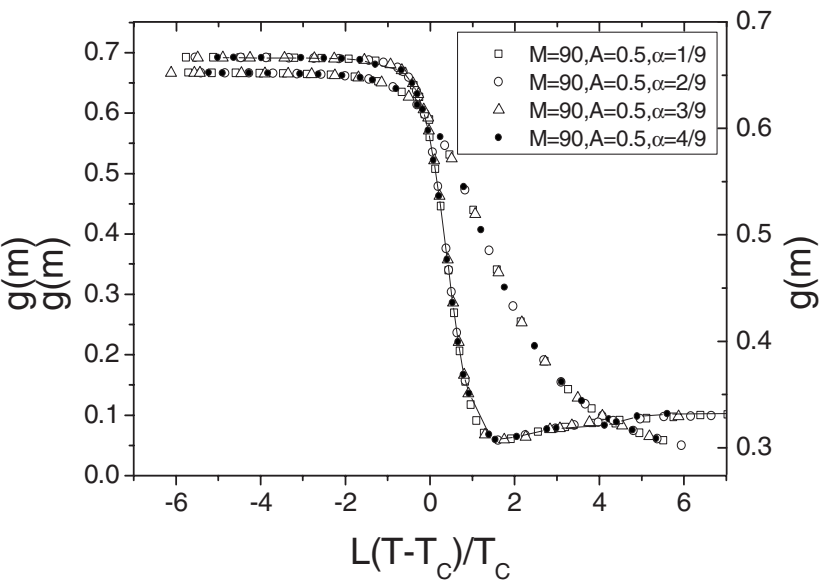

(b)

FIG. 4. The same plot as Fig. 3 for lattice structures with $A=$ (a) 1 and (b) 0.5 but different $\alpha$ values. 


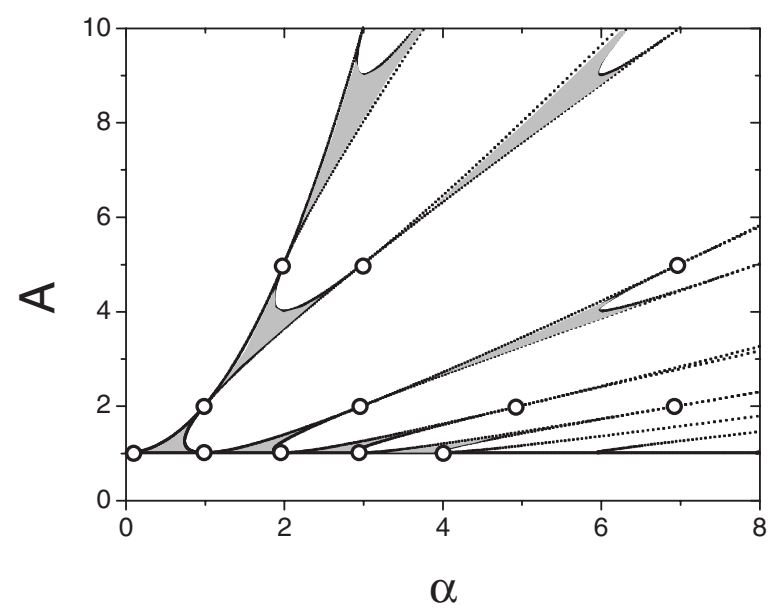

FIG. 5. Equivalent geometric structures possess the same FSSFs as $A=1$ in the plane of $A$ vs $\alpha$. The circles mark the loci of fully helical tori. The gray regions are $R_{2}$ and the others are $R_{1}$ as discussed in the text.

interiors of the circles of Fig. 6, while the intercircle domains pertain to the region $R_{2}$; the corresponding loci of fully helical tori remain on the intersections of the circles.

Our work has aimed at the classification of FSSFs for the geometry associated with general periodic BCs. Of particular interest is the appearance of self-similar pattern among different classes of FSSFs on the parametrization plane of geometry, and the pattern is shown to be the consequence of the transformation laws among equivalent geometric structures. In fact, the root cause for the ubiquity of fractal patterns in the natural world is the peculiar symmetries associated with

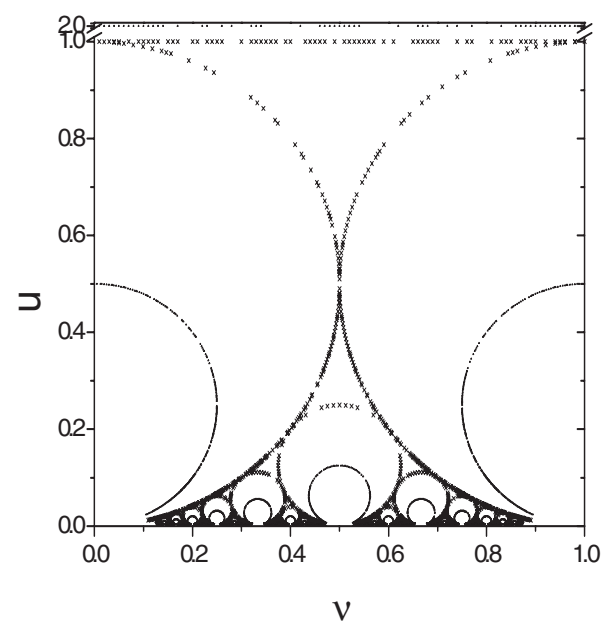

FIG. 6. Geometric structures in the plane of $u$ vs $v$ for two classes of FSSFs, with lines of crosses for $A=1$ and dotted lines for $A=0.5$. The circles mark the loci of fully helical tori.

the operations of simple transformation laws. Furthermore, the equivalence relations of geometric structures may also prove useful in enumerating the multiplicity of equivalence, subject to a variety of structural settings.

This work was partially supported by the National Science Council of Republic of China (Taiwan) under Grant No. NSC 95-2212-M-033-005 (M.C.H.) and the Alexander von Humboldt Foundation (Y.D.). We thank the National Center for High Performance Computing for providing the computing facilities.
[1] M. Fisher and M. N. Barber, Phys. Rev. Lett. 28, 1516 (1972).

[2] M. N. Barber, Phase Transitions and Critical Phenomena, edited by C. Domb and J. L. Lebowitz (Academic Press, New York, 1983), Vol. 8.

[3] Finite-Size Scaling, edited by J. L. Cardy (North-Holland, Amsterdam, 1988); Finite Size Scaling and Numerical Simulation of Statistical Systems, edited by V. Privman (World Scientific, Singapore, 1990).

[4] V. Privman, Phys. Rev. B 38, 9261 (1988).

[5] T. M. Liaw, M. C. Huang, S. C. Lin, and M. C. Wu, Phys. Rev. B 60, 12994 (1999); M.-C. Wu, C.-K. Hu, and N. Sh. Izmailian, Phys. Rev. E 67, 065103(R) (2003); M.-C. Wu, ibid. 73, 046135 (2006).

[6] Y. Okabe, K. Kaneda, M. Kikuchi, and C. K. Hu, Phys. Rev. E 59, 1585 (1999).

[7] R. M. Ziff, C. D. Christian, and P. Kleban, Physica A 266, 17 (1999).

[8] T. M. Liaw, M. C. Huang, Y. L Chou, S. C. Lin, and F. Y. Li, Phys. Rev. E 73, 055101(R) (2006).

[9] W. T. Lu and F. Y. Wu, J. Stat. Phys. 102, 953 (2001); W. Janke and R. Kenna, Phys. Rev. B 65, 064110 (2002); W. T. Lu and F. Y. Wu, Physica A 258, 157 (1998); Phys. Lett. A 259, 108 (1999).

[10] W. T. Lu and F. Y. Wu, Phys. Rev. E 63, 026107 (2001); K. Kaneda and Y. Okabe, Phys. Rev. Lett. 86, 2134 (2001).

[11] A. E. Ferdinand and M. Fisher, Phys. Rev. 185, 832 (1969).
[12] K. Binder, Z. Phys. B: Condens. Matter 43, 119 (1981); Phys. Rev. Lett. 47, 693 (1981).

[13] C.-K. Hu, J. Phys. A 27, L813 (1994); C.-K. Hu and J.-A. Chen, ibid. 28, L73 (1995); C.-K. Hu, C.-Y. Lin, and J.-A. Chen, Phys. Rev. Lett. 75, 193 (1995); 75, 2786(E) (1995); C.-K. Hu and C.-Y. Lin, ibid. 77, 8 (1996); Y. Okabe and M. Kikuchi, Int. J. Mod. Phys. C 7, 287 (1996); F.-G. Wang and C.-K. Hu, Phys. Rev. E 56, 2310 (1997).

[14] N. W. Ashcroft and N. Mermin, Solid State Physics (Harcourt Brace, New York, 1976).

[15] R. Sahito, G. Dresselhaus, and M. S. Dresselhaus, Physical Properties of Carbon Nanotubes (Imperial College Press, London, 1998).

[16] J. Cardy, Nucl. Phys. B 270 [FS16], 186 (1986); J.-B. Zuber, Phys. Lett. B 176B, 127 (1986).

[17] U. Wolff, Phys. Rev. Lett. 62, 361 (1989).

[18] F. Y. Wu, Rev. Mod. Phys. 54, 235 (1982).

[19] The definition of aspect ratio can be applied twofold. First, any of the toroidal $\mathrm{BCs}$ receives its own twisting prescription and is equipped with the conventional aspect ratio $A$. Second, the ratio of two radii intuitively defines the chiral aspect ratio $B$ for the fully helical tori [8], while the distinction between $B$ and $1 / B$ is insensitive to the FSSFs.

[20] B. Mandelbrot, The Fractal Geometry of Nature (W. H. Freeman and Co., New York, 1977). 